\title{
Correlation length of magnetosheath fluctuations: Cluster statistics
}

\author{
O. Gutynska, J. Šafránková, and Z. Němeček \\ Charles University, Faculty of Mathematics and Physics, V Holesovickach 2, 18000 Praha 8, Czech Republic
}

Received: 4 April 2008 - Revised: 17 June 2008 - Accepted: 30 June 2008 - Published: 1 September 2008

\begin{abstract}
Magnetosheath parameters are usually described by gasdynamic or magnetohydrodynamic (MHD) models but these models cannot account for one of the most important sources of magnetosheath fluctuations - the foreshock. Earlier statistical processing of a large amount of magnetosheath observations has shown that the magnetosheath magnetic field and plasma flow fluctuations downstream of the quasiparallel shock are much larger than those at the opposite flank. These studies were based on the observations of a single spacecraft and thus they could not provide full information on propagation of the fluctuations through the magnetosheath.

We present the results of a statistical survey of the magnetosheath magnetic field fluctuations using two years of Cluster observations. We discuss the dependence of the crosscorrelation coefficients between different spacecraft pairs on the orientation of the separation vector with respect to the average magnetic field and plasma flow vectors and other parameters. We have found that the correlation length does not exceed $\sim 1 R_{E}$ in the analyzed frequency range (0.001$0.125 \mathrm{~Hz}$ ) and does not depend significantly on the magnetic field or plasma flow direction. A close connection of crosscorrelation coefficients computed in the magnetosheath with the cross-correlation coefficients between a solar wind monitor and a magnetosheath spacecraft suggests that solar wind structures persist on the background of magnetosheath fluctuations.
\end{abstract}

Keywords. Magnetospheric physics (Magnetosheath)

\section{Introduction}

The magnetosheath is a region bounded at its outer edge by the bow shock and by the magnetopause at its inner edge. The importance of the magnetosheath lies in the fact that it

Correspondence to: J. Šafránková

(jana.safrankova@mff.cuni.cz) is the environment through which energy and momentum are transported from the solar wind into the Earth's magnetosphere. In the MHD description, the magnetosheath reconfigures the upstream solar wind flow and its frozen-in magnetic field to the state specified by the magnetopause boundary conditions. However, it is a simplification because this approach does not account for kinetic processes operating on small-scale lengths which also contribute to the plasma behavior.

Estimates of the global plasma properties in the magnetosheath are predominantly based on the results of the gasdynamic model predictions of Spreiter et al. (1966) and Spreiter and Stahara (1980). Their model assumes that bulk flow properties of the solar wind past a planetary obstacle can be described by the continuum equations of hydrodynamics for a single-component gas. A simplified non-self-consistent prescription for the magnetic field, which is frozen kinematically to the flow, means magnetic forces are omitted from the momentum equation.

In the model, the solar wind flows along the Sun-Earth line, strikes the subsolar magnetopause and then is diverted radially from this point. The model further predicts that velocities decrease from the bow shock to the magnetopause, whereas the density and temperature increase in the vicinity of the stagnation streamline. Farther from the subsolar region, the density and the velocity decrease but the temperature increases through radial profiles from the bow shock to the dayside magnetopause. Along the flanks of the nearEarth magnetotail, minimum velocities and maximum temperatures occur in the middle magnetosheath. The plasma flowing radially away from the stagnation streamline accelerates up to the solar wind speed and becomes increasingly like solar wind toward the flanks, where the bow shock is weaker. These predictions were generally confirmed by experimental studies but the gasdynamic approach cannot elucidate the problems of fluctuations of parameters because it allows only a single wave mode.

Zwan and Wolf (1976) used the results of the Spreiter et al. (1966) model at the bow shock and the magnetopause and

Published by Copernicus Publications on behalf of the European Geosciences Union. 
provided a formulation and numerical estimation of the magnetosheath flow using a MHD approach. Their model describes a magnetic flux tube moving from the bow shock to the magnetopause and predicted an increase of the magnetic field strength which is coupled with a plasma depletion. Both diversion of the flow at the bow shock along the magnetic field direction and a "squeezing" effect close to the magnetopause where flux tubes pile up are found to lead to a density depletion at the magnetopause. The result is a net density decrease in regions where the deceleration of the flow is not efficient and diversion of the flow dominates.

Southwood and Kivelson $(1992,1995)$ revisited the Zwan and Wolf (1976) model and proposed a solution for a few inconsistencies by adding a compressional front between the two depletion regions of the Zwan and Wolf (1976) model. This front compresses the plasma, while rarefying the magnetic field and diverts the plasma flow from the Earth-Sun line.

Wu (1992) made numerical simulations of the magnetosheath profile using a 3-D MHD calculation, taking into account the formation of a plasma depletion layer. In this model, the magnetosphere is a solid impermeable obstacle. The density increases first and then decreases from the bow shock toward the magnetopause along the Sun-Earth line. In the inner magnetosheath, the decrease with distance from the magnetopause is more abrupt than the increase in density within the outer magnetosheath.

Song et al. (1999a,b) have carried out comparisons of observations and MHD models. The authors discovered a region of a plasma density enhancement and magnetic field depression in the inner magnetosheath and attributed it to a slow-mode standing wave. Siscoe et al. (2002) discussed some aspects of the magnetosheath flow if magnetic forces are included in the framework of ordinary gasdynamics (Spreiter et al., 1966). The authors suggested four such aspects and illustrated them with computations using a numerical MHD code that simulates the global magnetosphere and its magnetosheath. Fuselier et al. (2002) compared observations of magnetosheath plasma in the high-altitude cusp with gas dynamic and MHD model predictions. They found that gasdynamic models over-estimate the flow velocity adjacent to the magnetopause at high latitudes while MHD models which include the effects of magnetic reconnection predicted lower flow velocities than those observed in the same region. However, some limitation of these comparisons is that they are either based on a single case study or a small number of observations.

Statistical studies of the magnetosheath structure and plasma parameters based on several years of observations in the flanks of the magnetosheath, both in the night and dayside, have been carried out using the Interball-1 data by Šafránková et al. (2004) and Němeček et al. (2000a,b, 2002a), using data from IMP 8 at the $-15<X_{\mathrm{GSE}}<-20 R_{E}$ slice and solar wind data from ISEE 1, ISEE 3 and WIND
(Paularena et al., 2001), and using four years of Cluster orbital coverage (Longmore et al., 2005, 2006).

These mentioned and other studies resulted in a consistent picture of average parameters in the near-Earth magnetosheath under various upstream conditions. However, magnetosheath magnetic field and ion flow fluctuations that are a significant phenomenon of the magnetosheath medium are still poorly understood, especially from a statistical point of view. In Zastenker et al. (1999), the middle-scale (from minutes to hours) variations of the ion flux and magnetic field magnitude were investigated in middle- and high-latitude magnetosheath regions near the terminator. The authors found that a portion of the observed variations originated in the subsolar region and propagated downstream with the magnetosheath speed. Zastenker et al. (2002) discussed the origin of magnetosheath variations and showed that a part of these variations is from propagation and/or amplification of solar wind or IMF disturbances which pass through the bow shock, and a part of these variations originates inside the magnetosheath. For very close (about $0.3 R_{E}$ ) spacecraft, the authors observed an absence of correlation for 5$20 \mathrm{~s}$ variations but a good correlation for about 3-min variations. On the other hand, they observed poor correlations between two well-separated (about $4 R_{E}$ ) spacecraft for 3$5 \mathrm{~min}$ variations but good correlations for longer (20-30 min) variations. The authors conclude that the persistence time of magnetosheath variations is roughly an order of magnitude larger than the period of the variations.

Luhmann et al. (1986) studied the spatial distribution of magnetosheath magnetic field fluctuations and showed that the amplitude of these fluctuations is controlled by the IMF direction and is larger behind quasi-parallel bow shocks. A comprehensive study of ion flux fluctuations in the magnetosheath (Němeček et al., 2002b) showed that the fluctuations are larger (1) behind the quasi-parallel bow shock, (2) closer to the magnetopause, and (3) during intervals of radial IMF. Moreover, they show that part of the magnetosheath fluctuations is of solar wind origin, whereas part is generated at the bow shock and/or in the magnetosheath proper (Zastenker et al., 2002).

A similar finding follows from the papers by Shevyrev and Zastenker (2005); Shevyrev et al. (2007). These authors concluded that in the magnetosheath, the plasma flow is mainly turbulent and that the character of the turbulence is strongly controlled by the $\theta_{B N}$ angle. Behind quasiparallel shocks, they observed different types of MHD-wave modes and variations of the ion flux and magnetic field increases. Behind quasiperpendicular bow shocks, they sometimes observed mirror-mode waves in the middle of the magnetosheath and near the magnetopause.

Blanco-Cano et al. (2006) performed global hybrid simulations and studied foreshock morphology and its influence on the bow shock and magnetosheath. The authors concluded that downstream from the shock, the magnetosheath is permeated by a variety of waves that result from the convection 
of upstream waves and also from local wave generation. The wave characteristics are different in the quasi-parallel and quasi-perpendicular parts of the magnetosheath.

Tatrallyay et al. (2002) identified mirror-type fluctuations in magnetic field data from the ISEE-1/2 spacecraft in different regions of the magnetosheath and concluded that these fluctuations do not always originate near the bow shock but that the source may be somewhere else (e.g. at the magnetopause, inside the magnetosheath, or in localized regions of the bow shock).

According to Lucek et al. (2001), the first results of mirror structure investigations based on four-point magnetic field observations by Cluster showed that these variations occur along the maximum variance direction on scales of $\sim 750$ $1000 \mathrm{~km}$. A detailed four-point Cluster study of mirror type magnetic field fluctuations by Tatrallyay et al. (2008) reveals that these fluctuations decrease in the inner regions of the magnetosheath, indicating some saturation in the growth of the waves when proceeding towards the magnetopause. The results suggest that mirror type fluctuations originate from the compression region downstream of the quasi-perpendicular bow shock and that the growth of the fluctuations cannot be described by linear approximations.

The direction of propagation of low-frequency waves (drift mirror and mirror mode waves) in the magnetosheath was studied by Narita and Glassmeier (2006). The authors found that the anti-sunward propagation (in the plasma frame) dominates. At small zenith angles, the propagation is toward the magnetosheath flank, and at large angles, it is toward the magnetopause. As a continuation of this study, Narita et al. (2006) conclude: the spatial pattern of wave propagation directions indicates that propagation is outwardly divergent in the upstream region, inwardly divergent in the magnetosheath near the subsolar magnetopause, and inwardly convergent at the magnetosheath flank. The divergent pattern in the magnetosheath indicates that the waves propagate along the plasma stream lines, following the refraction of the plasma flow at the shock. The convergent pattern in the magnetosheath flank is consistent with the perpendicular propagation in the draped magnetic field.

Recent analyses of solar wind-magnetosphere coupling use the measurements provided by the spacecraft in the solar wind. However, it is a magnetosheath plasma and magnetic field that come into a contact with magnetopause. There were a few attempts to compare the magnetosheath measurements with the observations at the magnetopause or in the magnetosphere but they showed only qualitative results (e.g. Měrka et al., 2000). The problem is connected with the magnetosheath fluctuations. They can significantly change the conditions at the magnetopause because their amplitudes are often larger than the mean value of a corresponding parameter. At present, there are several spacecraft that can be used as magnetosheath monitors (e.g. Cluster, Themis, Geotail) for particular studies, but it is not clear how far the monitor can be for a reliable prediction of the parameters at the stud-

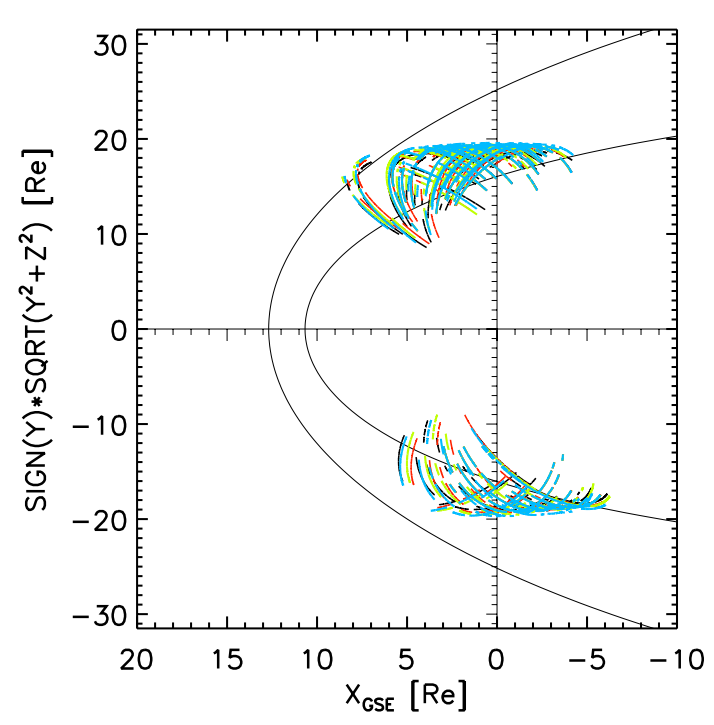

Fig. 1. Radial projections of four Cluster spacecraft orbits. The data used are from the intervals of May-June, 2002, 2003 and from November and December, 2002, 2003. The full lines display average locations of both magnetopause and bow shock determined from the Petrinec and Russell (1996) and Jeřáb et al. (2005) models, respectively.

ied interaction region. In other words, the correlation length of magnetosheath fluctuations is generally unknown.

In this paper, we perform a statistical analysis of the correlation length of magnetosheath magnetic field fluctuations near the dawn-dusk meridional plane. For our preliminary study, we chose the magnetic field strength because magnetic field measurements are available from all Cluster spacecraft. This preference implies that the analysis is limited to compressible fluctuations, but the problem of variations of the magnetic field direction is more complex and we will return to it in a consecutive study.

Our investigation is restricted to the frequency range from 0.001 to $0.125 \mathrm{~Hz}$. The study reveals several surprising facts: the correlation length of analyzed variations is as short as $1 R_{E}$ and this length does not depend significantly on the direction of the magnetic field or plasma flow. On the other hand, for a particular range of spacecraft separations in the magnetosheath, the cross-correlation coefficient increases with the cross-correlation between magnetosheath and solar wind observations.

\section{Magnetosheath data coverage and their selection}

As stated above, the analysis is based on the magnetic field strength measured by four Cluster spacecraft in the magnetosheath near the dawn-dusk meridional plane. The reason for this selection was that Cluster spent usually several hours 


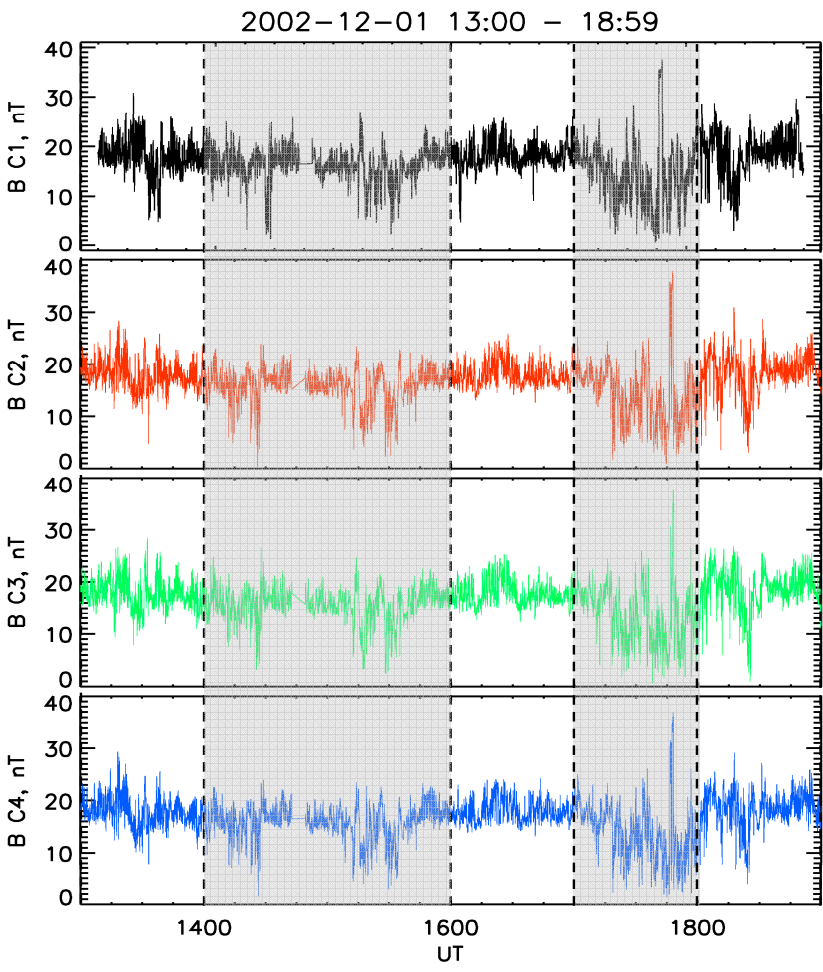

Fig. 2. An example of magnetosheath magnetic field strength measurements made by all Cluster spacecraft on 12 December 2002 and the way of data selection. Two shadowed intervals denote those intervals that were excluded from data processing.

continuously in the sheath region and provided a sufficient amount of data for statistical processing.

The radial projection of the orbits used for analysis is shown in Fig. 1 together with the average model bow shock (Jeřáb et al., 2005) and magnetopause (Petrinec and Russell, 1996) positions. Since the Cluster apogee is low, a majority of data was collected in the magnetopause vicinity. A part of the orbits seems to lie outside of the magnetosheath in Fig. 1, however, this impression is caused by uncertainty in the applied models and variability of upstream parameters. We have carefully chosen only the intervals when all Cluster spacecraft were located in the magnetosheath. The selection of the intervals proceeded in several steps. In the first step, we selected four basic intervals (May-June and NovemberDecember 2002 and 2003) when the Cluster spacecraft were orbiting in our chosen region. The second step consisted of a visual inspection of daily plots of plasma and magnetic field parameters and a rough identification of the magnetosheath intervals. These intervals were divided into subintervals of 60-min length and each of them underwent a new inspection to discard the subintervals containing bow shock or magnetopause crossings or data gaps. An example of our selected data is shown in Fig. 2. This figure presents $6 \mathrm{~h}$ of magnetosheath observations on 12 December 2002 from 13:00 to
19:00 UT. The shadowed intervals were excluded from further processing, from 14:00 to 16:00 UT due to data gaps, and from 17:00 and 18:00 UT due to the magnetopause crossing at $\sim 17: 50$ UT in data of all Cluster spacecraft. Figure 2 shows only the magnetic field magnitude, but we checked that the large increase of the magnetic field in this time corresponds to the spacecraft exit into the magnetosphere.

Altogether, we selected $\sim 740$ one-hour intervals for further computation. Since our statistics are limited to lowfrequency fluctuations that have enough power to influence the magnetosheath-magnetosphere coupling, we have used spin-averaged data. Their temporal resolution is approx. $4 \mathrm{~s}$, similar for all spacecraft. These data were used without any further processing for computation of standard deviations that stand as a measure of the fluctuation power.

The cross-correlation function, $r$, was computed according to the formula:

$$
r=\frac{\frac{1}{N-1} \sum_{i=0}^{N-1}\left(x_{i}-\sum_{k=0}^{N-1} \frac{x_{k}}{N}\right)\left(y_{i}-\sum_{k=0}^{N-1} \frac{y_{k}}{N}\right)}{\sqrt{\frac{1}{N-1} \sum_{i=0}^{N-1}\left(x_{i}-\sum_{k=0}^{N-1} \frac{x_{k}}{N}\right)^{2}} \sqrt{\frac{1}{N-1} \sum_{i=0}^{N-1}\left(y_{i}-\sum_{k=0}^{N-1} \frac{y_{k}}{N}\right)^{2}}}
$$

where $x_{i}, y_{i}$ stands for two $N$-element magnetic field samples from two Cluster spacecraft. This formula requires the data measured in equidistantly spaced times that are identical for all spacecraft. Since the study is based on spin averaged data and the spin periods of Clusters are different, an unified time scale was applied and the data of all spacecraft were linearly approximated (truncated) to this scale for estimation the correlation functions.

The cross-correlation functions were computed on 1200-s intervals in the middle of each 1-h subintervals with the lag ranging from -1200 to $+1200 \mathrm{~s}$. In order to reflect its limited duration and to hold the Nyquist criterion, the data were passed through band-pass filter $0.001-0.125 \mathrm{~Hz}$ prior the correlation. The cross-correlation functions computed for all six pairs of the Cluster spacecraft over the time interval 13:00 14:00 UT (the first interval in Fig. 2) are presented in Fig. 3 as examples. One can note a very good $(\sim 0.8)$ correlation between the $\mathrm{C} 2$ and $\mathrm{C} 4$ spacecraft, whereas all others are at a level of $\sim 0.25$ in spite of the fact that the correlation between C3 and C4 peaks for a lag equal to zero. Since the correlation between two magnetosheath points can be a function of many parameters, we will analyze it statistically. However, prior to further analysis, we should ensure that our selection is not biased in any respect. For this reason, we present histograms of several basic parameters in the following figures. Since the dawn observations were carried out in the summer, whereas the dusk sector was covered by Cluster at the end of each year, we present the distributions of the parameters for the dawn and dusk sectors separately.

Probably the most important factor that can influence the cross-correlation is the spacecraft separation. Figure 4 shows 


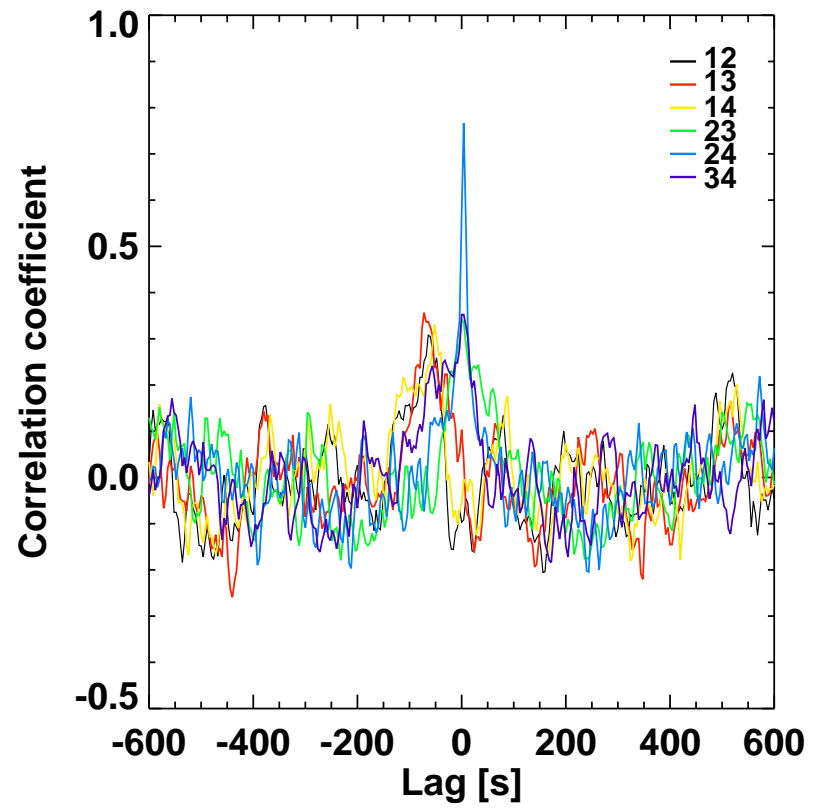

Fig. 3. An example of cross-correlation functions computed between Cluster spacecraft pairs (denoted different colors) between 13:00 and 14:00 UT, 12 December 2002 (only $\pm 600 \mathrm{~s}$ of the lag range is shown).

the number of observed events as a function of the spacecraft separation. Note that, since the Cluster project prefers short separations, we applied non-linear scales. Nevertheless, our set contains several events with separations exceeding $4 R_{E}$. In the figure, the colors distinguish dawn and dusk intervals and one can see that the number of events is similar for both flanks up to $2.5 R_{E}$ and larger separations occurred only for the dusk intervals.

\section{Upstream conditions}

The values of the plasma parameters measured in the magnetosheath depend on the upstream solar wind conditions. We applied ACE (Smith et al., 1998; McComas et al., 1998) and Wind (Ogilvie et al., 1995; Lepping et al., 1995) as monitors of solar wind and IMF data. We found a slightly better organization of magnetosheath fluctuation measurements using Wind observations. The reason probably is that Wind is usually located closer to Earth. The following series of figures shows the Wind data lagged by the estimated propagation time and averaged over the duration of the analyzed intervals (1 h).

The figures present upstream conditions under which the study was performed. The distribution of solar wind speeds in Fig. 5a shows that a great majority of events occurs during periods of slow solar wind; the secondary peak corresponding to fast solar wind streams $(\sim 700 \mathrm{~km} / \mathrm{s})$ is about five times

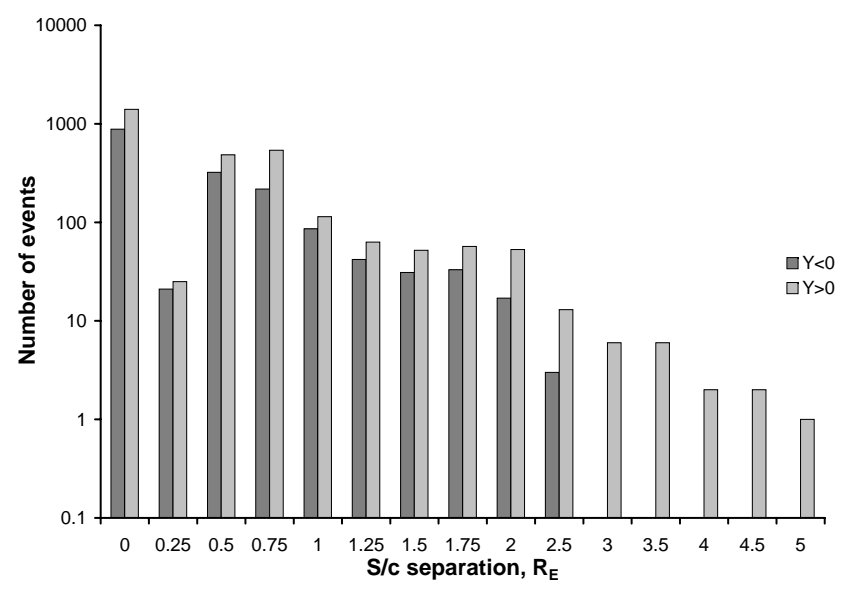

Fig. 4. Distributions of spacecraft separations in the analyzed set for dusk and dawn subsets. Note that both vertical and horizontal scales are non-linear.

lower. This result is consistent with the fact that the analyzed period lies in the descending part of the solar cycle. We think that a larger number of events with very a slow $(<400 \mathrm{~km} / \mathrm{s})$ solar wind velocity for dawn events is probably caused by already mentioned seasonal difference between dusk and dawn observations - the dusk observations are a half of the year closer to solar minimum. The distribution of solar wind densities in Fig. 5b is consistent with Fig. 5a because the solar wind velocity and density usually are anti-correlated.

The histograms of upstream magnetic field strength and components are shown in Fig. 6. We see a typical IMF distribution with the peak at $\sim 6 \mathrm{nT}$ for both dawn and dusk sets in Fig. 6a. The next two panels (Fig. 6b, c) exhibit two-peak distributions of IMF horizontal components. These distributions are connected with a prevailing IMF orientation along the Parker spiral that causes the dawn magnetosheath being more frequently behind the quasiparallel shock. Nevertheless, a non-negligible number of events with very small horizontal IMF components stresses the importance of IMF $B_{Z}$, shown in the last panel (Fig. 6d). Its importance is underlined by the fact that a large portion of the observations was made in the magnetopause vicinity (Fig. 1). A brief inspection in Fig. 6d reveals a peak at $B_{Z}=0$ and a slight preference for negative values that is similar for the dawn and dusk sets. Finally, we conclude that the distributions in Figs. 5 and 6 resemble typical solar wind features and that there is no bias toward extreme values of upstream parameters in our selection. This is true for both the dawn and dusk subsets and any potential difference between flanks can be determined reliably. 


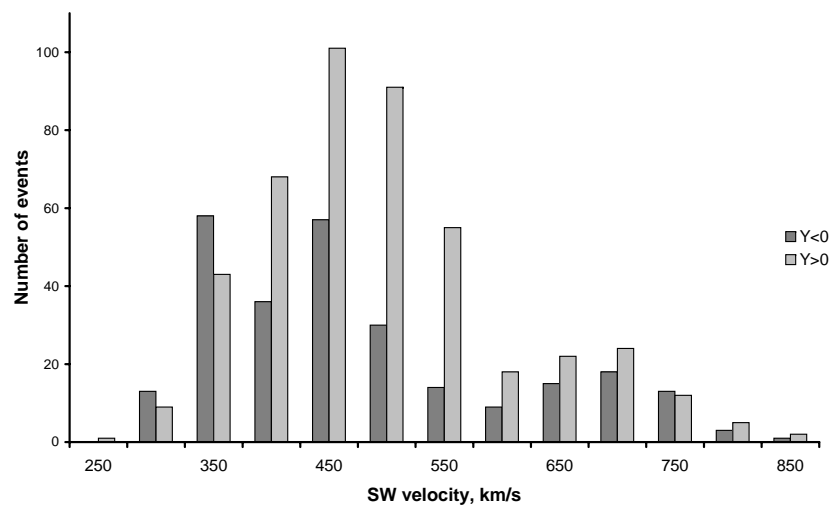

(a)

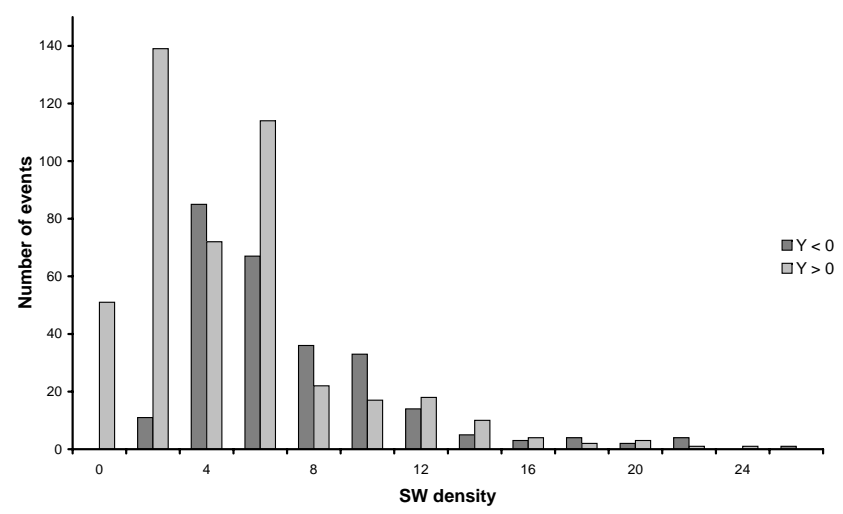

(b)

Fig. 5. Histograms of averaged solar wind velocities (a) and densities (b) during selected magnetosheath intervals.

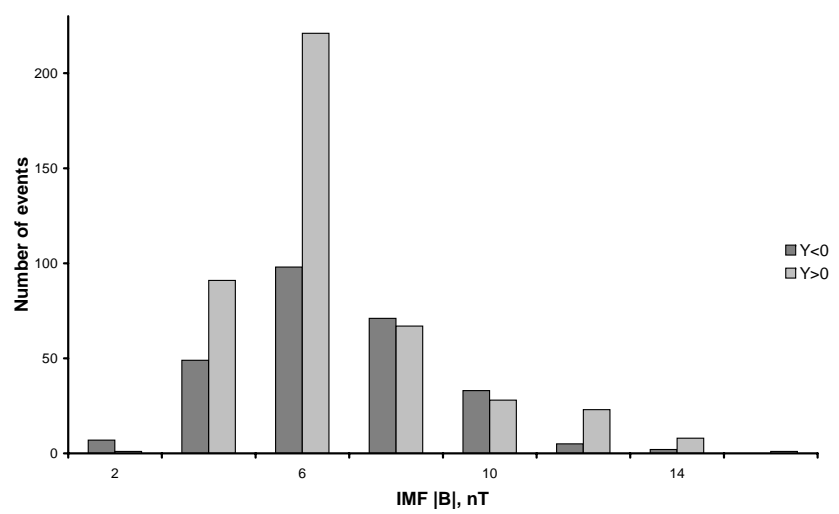

(a)

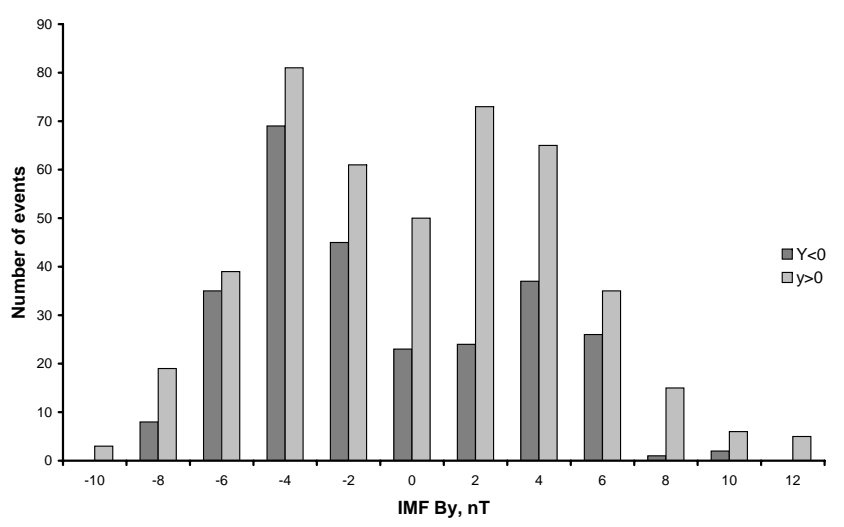

(c)

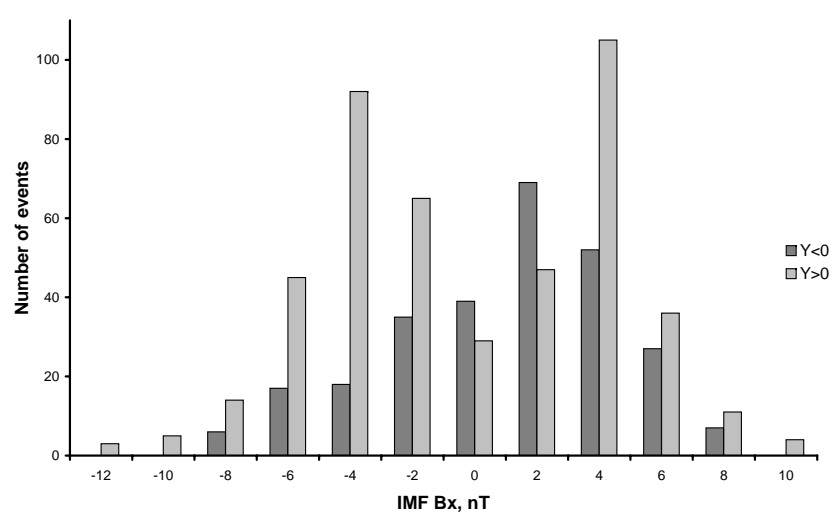

(b)

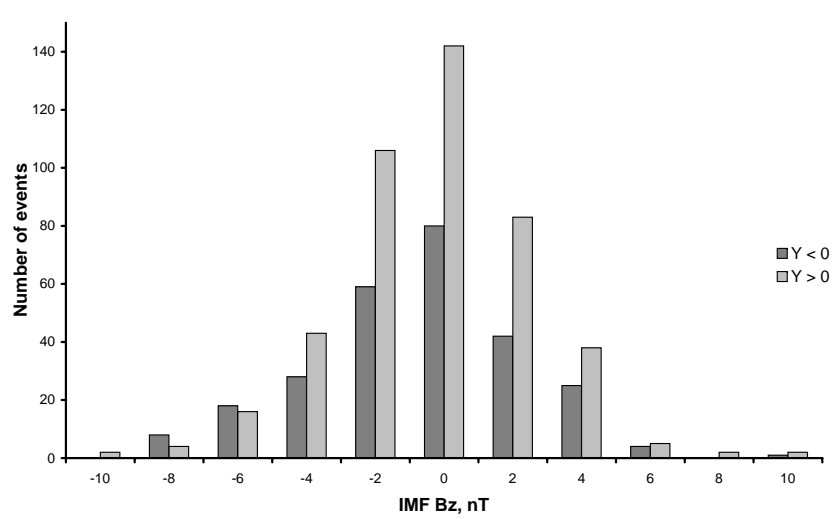

(d)

Fig. 6. Histograms of averaged magnetic field strength (a) and three (b-d) components for the events at the dusk and dawn magnetosheath (in the GSE coordinate system).

\section{Correlation length of magnetic field fluctuations}

Four Cluster spacecraft create six pairs and thus provide $\sim 4500$ correlation coefficients for determination of the correlation length of magnetic filed fluctuations. Although the magnetometers onboard the spacecraft are identical, we checked if these coefficients can be used regardless of composition of a particular spacecraft pair. We used the shortest separations since in that case, the difference (if any) among various $\mathrm{s} / \mathrm{c}$ pairs would be mostly caused by a difference in 


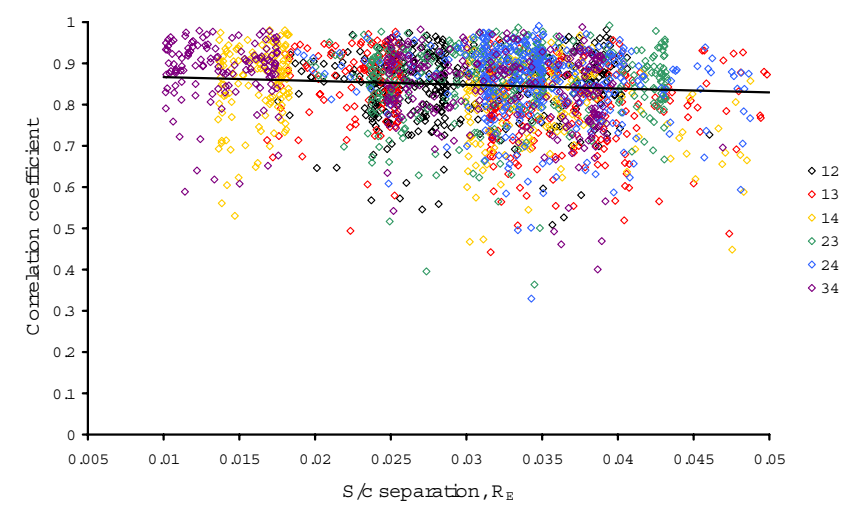

Fig. 7. Cross-correlation coefficients as a function of the s/c separation. Only the separations shorter than $0.05 R_{E}$ are displayed, the different colors denote a spacecraft pair (see a legend on the right side of the figure).

the measuring equipment. Figure 7 shows the correlation coefficients as a function of the spacecraft separation. Since the correlation function depends on the time lag, the maxima are plotted in this and all following figures. The different spacecraft pairs are distinguished by the colors and only separations less than $0.05 R_{E}$ are shown. Since no systematic difference between the s/c pairs can be observed, we do not distinguish the s/c pair used for computation of a particular correlation coefficient in our analysis. However, the figure reveals one striking feature of magnetosheath variations - the correlation coefficient can be as low as 0.5 for points separated by $100 \mathrm{~km}$. The wave length of plasma waves in our frequency range is much larger $(>1000 \mathrm{~km})$. The linear approximation of the trend shown by the heavy line leads to a value of about $1 R_{E}$ for a correlation coefficient equal to zero. We are using this way for a definition of the correlation length instead the fitting with an exponential function because the values of correlation coefficients depend on the number of samples used for correlation $(\sim 300$ in our case); this effect is more pronounced at larger separations. We will discuss this dependence later.

A similar result can be derived from Fig. 8, where average values of correlation coefficients (regardless of the spacecraft pair) are plotted for the whole data set. The bins for averaging increase with the s/c separation in order to obtain a reasonable number of events in each bin (compare with Fig. 4). The averaged correlation coefficient rapidly falls down with the s/c separation until $\sim 0.2 R_{E}$ and it exhibits a saturation at a level of $\sim 0.3$ for the largest s/c separations. The trend can be described rather well by a power law in a form $y=A \cdot x^{B}$ (see the heavy line in the figure), so we use this fit in our analysis.

The influence of the foreshock can be estimated by dividing the data set into dawn and dusk subsets. The result is plotted in Fig. 9; the different flanks are distinguished by the col-

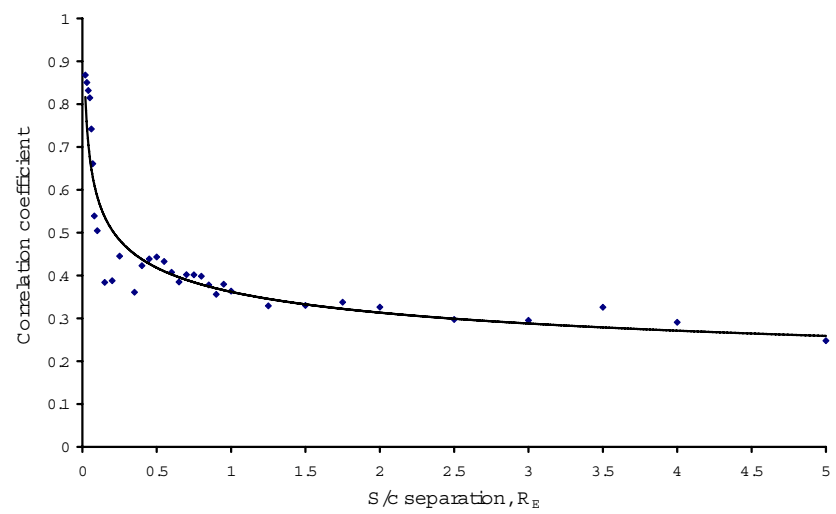

Fig. 8. Averages of cross-correlation coefficients as a function of the s/c separation for a full set of events. The heavy line stands for a power-law fit.

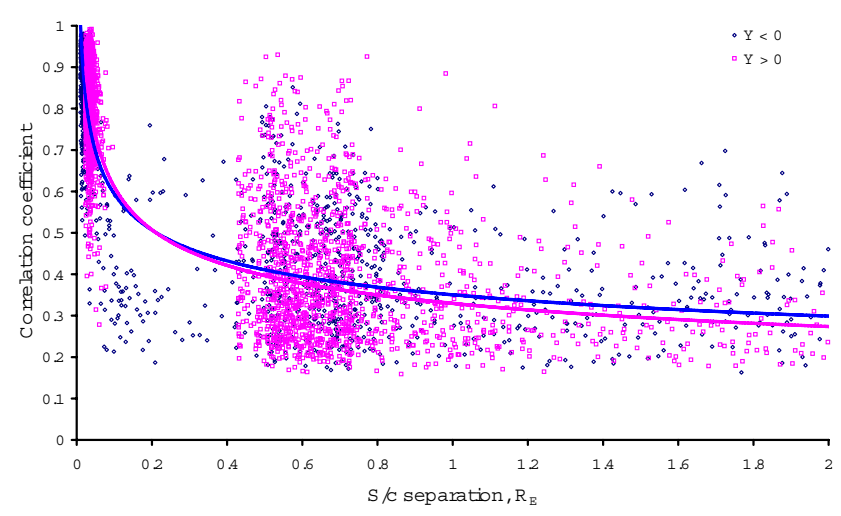

Fig. 9. Cross-correlation coefficients plotted vs the s/c separation for the dawn and dusk magnetosheaths. A full set of the events and power-law fits are presented up to $2 R_{E}$ of s/c separations.

ors. The horizontal axis is limited to $2 R_{E}$ because there are no data for larger s/c separations in the dusk magnetosheath (Fig. 4). The distribution of points as well as the fits are very similar for both flanks. Taking into account the spread of points, the difference between fits is not statistically significant and the presence of the foreshock fluctuations does not influence the correlation length in the magnetosheath.

The magnetosheath fluctuations are often treated as an ensemble of plasma waves, thus the direction of the magnetic field with respect to the s/c separation vectors would influence the value of the correlation coefficient. However, such investigation should reflect the dependence of the correlation coefficient on the separation distance shown in previous figures. Following the profile of this dependence, we have divided our set into three subsets according to separation length: separations shorter than $0.4 R_{E}$, separations between 0.4 and $1 R_{E}$ and larger separations (i.e. $>1 R_{E}$ ). The reasons for the choice of these break points are seen in 


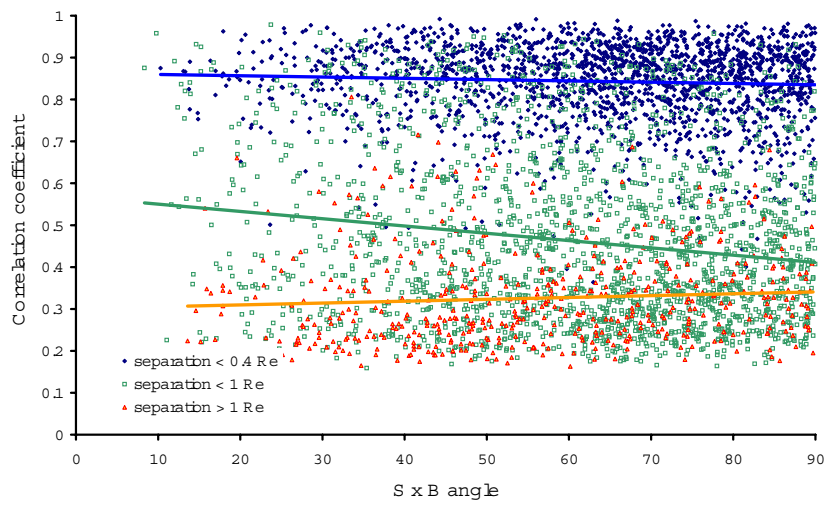

(a)

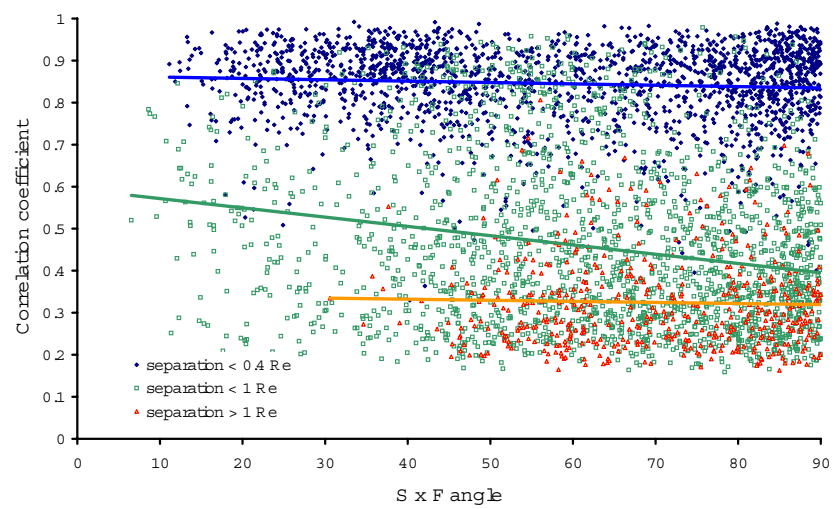

(b)

Fig. 10. Cross-correlation coefficients as a function of the angle between the s/c separation and averaged magnetic field vectors, $S \times B($ a) and the angle between the s/c separation and ambient plasma velocity vectors, $S \times F(\mathbf{b})$. The events are grouped according to the length of the $\mathrm{s} / \mathrm{c}$ separation and linear fits are shown for each group.

Fig. 9. In our set, we have a large group of short separations and the other group for separations larger than $0.4 R_{E}$. The $1 R_{E}$ break point was chosen because we determined the correlation length of this order and we think that the behavior of correlation coefficients can be different for larger separations.

Figure 10a presents the correlation coefficient as a function of the angle between the separation vector and averaged magnetic field ( $S \times B$ angle) for the above groups of events. The data belonging to each of these groups are fitted with a linear fit. The group of shortest separations exhibits only a weak dependence of the correlation coefficient on the $S \times B$ angle. This effect can be expected because a typical correlation in this group is $\sim 0.85$. Nevertheless, the correlations are larger for small angles. This trend can be clearly seen in the second group (separations between 0.4 and $1 R_{E}$ ) but the trend is very weak and reversed in the group of largest separations. However, a usual value of the correlation coefficients in this group is only $\sim 0.3$ and we will discuss the meaning of such low correlations later. We conclude that the correlations are slightly larger for the direction parallel to the magnetic field. The waves propagating along the ambient magnetic field are correlated over longer distances than those proceeding in the perpendicular direction.

Magnetosheath fluctuations are expected to be blown downward with the plasma flow (e.g. Hayosh et al., 2005) and thus the correlation length would be larger along the velocity direction. The results of our analysis are shown in Fig. $10 \mathrm{~b}$ where the correlation coefficients are plotted vs the angle between the s/c separation and velocity vectors $(S \times F$ angle). This angle is in the range $0-90^{\circ} ; 0^{\circ}$ stands for either parallel or antiparallel orientation of the vectors. Since the directions of the separation vectors are distributed randomly, we do not distinguish between these two cases because the change of a sign of the separation vector changes the sign of the lag but not the peak value of the correlation coefficient. Figure 10b shows larger correlations for small $S \times F$ angles for all groups of s/c separations. Similarly to the previous figure, this trend is very clear for the separations between 0.4 and $1 R_{E}$ and weak for other two groups. We note that we used the instantaneous values of the magnetic field and velocity components measured by Cluster 3 for a computation of the angles and than we averaged them over $20 \mathrm{~min}$. The background directions of the magnetic field and plasma velocity differ at locations of different spacecraft, but we think that this fact cannot spoil our analysis because we investigate the flank magnetosheath where the draping effect is not so pronounced and reasonable correlations were obtained for separations shorter than $1 R_{E}$.

As Zastenker et al. $(1999,2002)$ noted, the magnetosheath variations can be divided into two classes: variations penetrating through the bow shock from the solar wind and intrinsic magnetosheath variations. We checked whether these two classes behave the same way using the correlation of WIND and $\mathrm{C} 1$ magnetic fields as a measure of penetration of solar wind variations into the magnetosheath. Figure 11 shows the correlation coefficients between the Cluster pairs as a function of the C1-WIND correlation coefficients. The data are again sorted according to the s/c separations. The conclusion from this figure is clear; the correlation between two magnetosheath points increases with the correlations of IMF and magnetosheath magnetic field regardless of the s/c separations. A larger magnetosheath-solar wind correlation can probably be interpreted as an increasing portion of solar wind variations in the magnetosheath and the trends in Fig. 11 suggest that these variations correlate over longer distances in the magnetosheath than intrinsic magnetosheath fluctuations. 


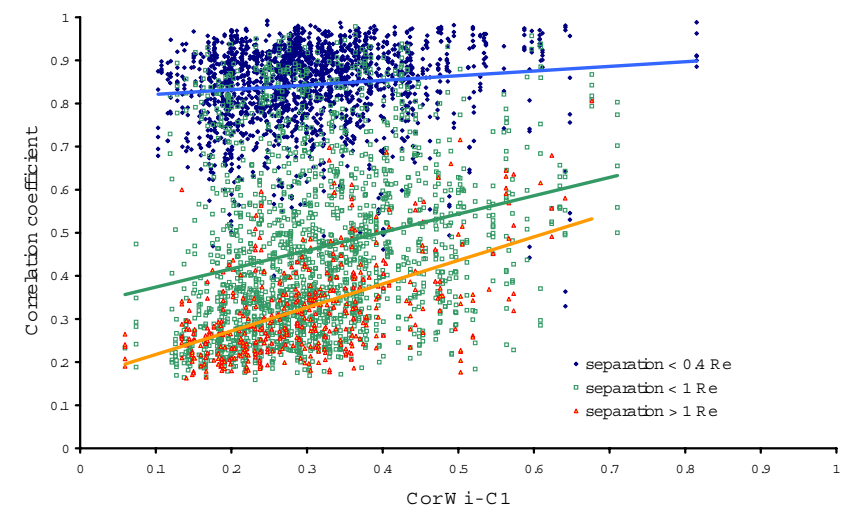

Fig. 11. Cross-correlation coefficients between two magnetosheath points as a function of the cross-correlation function between IMF (Wind) and magnetosheath magnetic field. See the text for a detailed description.

\section{Discussion}

Our analysis of two years of the Cluster magnetic field measurements in the vicinity of the dawn-dusk meridional plane has shown that the correlation length of the fluctuations in the range of $0.001-0.125 \mathrm{~Hz}$ is approximately $1 R_{E}$ in a statistical sense. This value is consistent with that obtained by Lucek et al. (2001) in their case study of mirror mode waves. However, we found a number of cases when the correlation falls to $0.5-0.6$ for separations shorter than $0.05 R_{E}$. If the fluctuations are small, their correlation may be poor because the contribution of the natural and uncorrelated noise prevails. Thus, we calculated standard deviations along all analyzed intervals and we show them in Fig. 12. The standard deviations range from 1 to $16 \mathrm{nT}$ (the background field was $10-40 \mathrm{nT})$. When the separation is short, the standard deviations are nearly the same at all Cluster spacecraft. Significant differences can be found only for large separations $\left(>1 R_{E}\right)$ and the largest fluctuations $(S D>10)$.

In order to check the influence of the fluctuation strength on our results, we repeated the analysis shown in Figs. 711 with the half of the data exhibiting the largest standard deviations $(S D>3.5 \mathrm{nT})$; the results were exactly the same.

Another problem is that we compute the correlation coefficients from discrete data on a limited time interval. The theory of a correlation function computed for one realization of a process expects: (1) the process is stationary and (2) an unlimited time interval is available. The solar wind and IMF cannot be considered as stationary on any time scale, but this problem can be significantly reduced by using an appropriate filter as we do. In order to estimate the influence of the limited number of points used for the calculation, we generated several random sequences that simulate the Cluster data and applied the same procedures for calculation of correlations. When we use 300 points (a number of points used for corre-

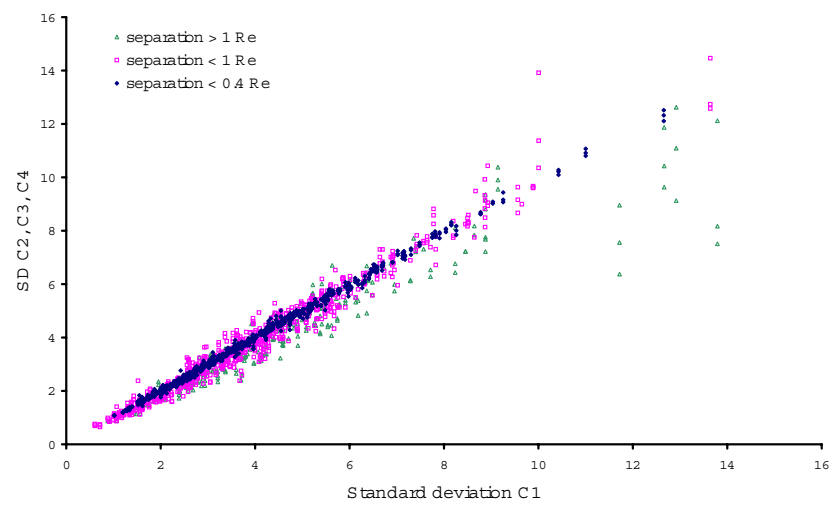

Fig. 12. A scatter plot of standard deviations of $\mathrm{C} 2, \mathrm{C} 3$, and $\mathrm{C} 4$ magnetic field measurements as a function of standard deviations of $\mathrm{C} 1$ measurements for three groups of s/c separations.

lations in this paper), the correlation coefficients ranged from 0.16 to 0.22 , whereas using five times as many points leads to coefficients that do not exceed 0.1. Based on this analysis, we conclude that the correlation coefficients on a level of 0.25 means that the signals are essentially uncorrelated. This suggests that the trend of the plots of correlation coefficients vs the s/c separation to saturation at a level of $\sim 0.25$ is a product of the data processing but it does not change our conclusion that a typical correlation length of the magnetosheath fluctuations is about $1 R_{E}$. As we noted above, this length is shorter than the wave length of the waves in the considered frequency range. On the other hand, many studies show that the waves of different modes can propagate (or be blown with the magnetosheath flow) over large distances. However, these studies dealt with a particular wave mode and we are dealing with the full ensemble of waves in our frequency range. Since the magnetosheath plasma is highly dispersive, these waves propagate with different phase speeds. Moreover, the phase speed of a particular wave changes in space and time due to a presence of other waves that modulate the background parameters. Consequently, the large values of correlation coefficients for separations in excess of $1 R_{E}$ can be achieved when some wave mode and frequency dominate or in the case of strong external (upstream) perturbations. We assume that this fact can explain the profile of correlation coefficients for larger separations but the reason for very low correlations at short separations remains unknown.

\section{Conclusion}

We statistically analyzed two years of the Cluster magnetosheath magnetic field measurements in a vicinity of the dawn-dusk meridional plane. We summarize that

- The correlation length of the magnetosheath magnetic field variations is approx. $1 R_{E}$. 
- This correlation length only weakly depends on the direction of the ambient magnetic field or the plasma flow, in both cases being slightly larger for parallel separations.

- The variations penetrating from the solar wind exhibit a larger correlation length than the intrinsic magnetosheath fluctuations.

The last point is important for predicting magnetopause processes bases on the solar wind monitoring. However, further study is required to quantify this result.

Acknowledgement. The authors thank the FGM Cluster team for the use of magnetic field data. The present work was partly supported by the Czech Grant Agency under Contracts 205/06/0875, 205/07/0694, and 202/08/H057, partly by the Research Plan MSM 0021620860 that is financed by the Ministry of Education of the Czech Republic and partly by the INTAS project $05-100000-8$ 8050. O. Gutynska thanks to the Grant Agency of the Charles University for support via project No. 147307.

Topical Editor R. Nakamura thanks two anonymous referees for their help in evaluating this paper.

\section{References}

Blanco-Cano, X., Omidi, N., and Russell, C. T.: Macrostructure of collisionless bow shocks: 2. ULF waves in the foreshock and magnetosheath, J. Geophys. Res., 111(A10), A10205, doi:10.1029/2005JA011421, 2006.

Fuselier, S. A., Waite, J. H., Avanov, L. A., Smirnov, V. M., Vaisberg, O. L., Siscoe, G., and Russell, C. T.: Characteristics of magnetosheath plasma in the vicinity of the high-altitude cusp, Planet. Space Sci., 50, 559-566, 2002.

Hayosh, M., Šafránková, J., Němeček, Z., Přech, L., Kudela, K., and Zastenker, G. N.: Relationship between high-energy particles and ion flux in the magnetosheath, Planet Space Sci., 53(13), 103-115, 2005.

Jeřáb, M., Němeček, Z., Šafránková, J. , Jelínek, K., and Merka, J.: A study of bow shock locations, Planet. Space Sci., 53, 85-94, 2005.

Lepping, R. P., Acuna, M. H., Burlaga, L. F., et al.: The Wind magnetic field investigation, Space Sci. Rev., 71, 207-229, 1995.

Longmore, M., Schwartz, S. J., Geach, J., Cooling, B. M. A., Dandouras, I., Lucek, E. A., and Fazakerley, A. N.: Dawn-dusk asymmetries and sub-Alfvenic flow in the high and low latitude magnetosheath, Ann. Geophys., 23, 3351-3364, 2005, http://www.ann-geophys.net/23/3351/2005/.

Longmore, M., Schwartz, S. J., and Lucek, E. A.: Rotation of the magnetic field in Earth's magnetosheath by bulk magnetosheath plasma flow, Ann. Geophys., 24, 339-354, 2006, http://www.ann-geophys.net/24/339/2006/

Lucek, E. A., Dunlop, M. W., Horbury, T. S., Balogh, A., Brown, P., Cargill, P., Carr, C., Fornacon, K.-H., Georgescu, E., and Oddy, T.: Cluster magnetic field observations in the magnetosheath: four-point measurements of mirror structures, Ann. Geophys., 19, 1421-1428, 2001,

http://www.ann-geophys.net/19/1421/2001/.
Luhmann, J. G., Russell, C. T., and Elphic, R. C.: Spatial distributions of magnetic field fluctuations in the dayside magnetosheath, J. Geophys. Res., 91, 1711-1715, 1986.

McComas, D. J., Bame, S. J., Barker, P., et al.: Solar Wind Electron Proton Alpha Monitor (SWEPAM) for the Advanced Composition Explorer, Space Sci. Rev., 86(1-4), 563-612, 1998.

Měrka, J., Šafránková, J., Němeček, Z., Savin, S., and Skalsky, A.: High-altitude cusp: INTERBALL observation, Adv. Space Res., 25(7-8), 1425-1434, 2000.

Narita, Y. and Glassmeier, K.-H.: Propagation pattern of low frequency waves in the terrestrial magnetosheath, Ann. Geophys., 24, 2114-2444, 2006, http://www.ann-geophys.net/24/2114/2006/.

Narita, Y., Glassmeier, K. H., Fornacon, K. H., Richter, I., Schafer, S., Motschmann, U., Dandouras, I., Reme, H., and Georgescu, E.: Low-frequency wave characteristics in the upstream and downstream regime of the terrestrial bow shock, J. Geophys. Res., 111, 1636, doi:10.1029/2005JA011231, 2006.

Němeček, Z., Šafránková, J., Přech, L., Zastenker, G. N., Paularena, K. I., and Kokubun, S.: Magnetosheath study: INTERBALL observations, Adv. Space Res., 25, 1511-1516, 2000a.

Němeček, Z., Šafránková, J., Zastenker, G. N., Pišoft, P., Paularena, K. I., and Richardson, J. D.: Observations of the radial magnetosheath profile and a comparison with gasdynamic model predictions, Geophys. Res. Lett., 27, 2801-2804, 2000b.

Němeček, Z., Šafránková, J., Zastenker, G., Pišoft, P., and Jelínek, $\mathrm{K}$.: Low-frequency variations of the ion flux in the magnetosheath, Planet. Space Sci., 50, 567-575, 2002a.

Němeček, Z., Šafránková, J., Zastenker, G. N., Pišoft, P., and Paularena, K. I.: Spatial distribution of the magnetosheath ion flux, Adv. Space Res., 30(12), 2751-2756, 2002b.

Ogilvie, K. W., Chornay, D. J., Fritzenreiter, R. J., et al.: SWE: A comprehensive plasma instrument for theWind spacecraft, Space Sci. Rev., 71, 55-77, 1995.

Paularena, K. I., Richardson, J. D., Kolpak, M. A., Jackson, C. R., and Siscoe, G. L.: A dawn-dusk density asymmetry in Earth's magnetosheath, J. Geophys. Res., 106, 25 377-25 394, 2001.

Petrinec, S. M. and Russell, C. T.: Near-Earth magnetopause shape and size as determined from the magnetopause flaring angle, $\mathrm{J}$. Geophys. Res., 101, 137-152, 1996.

Šafránková, J., Hayosh, M., Němeček, Z., and Přech, L.: Magnetosheath investigations: Interball contribution to the topic, in: Multiscale processes in the Earths Magnetosphere: From Interball to Cluster, 73-94, Kluwer Academic Publishers, 2004.

Shevyrev, N. N. and Zastenker, G. N.: Some features of the plasma flow in the magnetosheath behind quasi-parallel and quasi-perpendicular bow shocks, Planet. Space Sci., 53, 95-102, 2005.

Shevyrev, N. N., Zastenker, G. N., and Du, J.: Statistics of low-frequency variations in solar wind, foreshock and magnetosheath: INTIERBALL-1 and CLUSTER data, Planet. Space Sci., 55(15), 2330-2335, 2007.

Siscoe, G. L., Crooker, N. U., Erickson, G. M., Sonnerup, B. U. O., Maynard, N. C., Schoendorf, J. A., Siebert, K. D., Weimer, D. R., White, W. W., and Wilson, G. R.: MHD properties of magnetosheath flow, Planet. Space Sci., 50, 461-471, 2002.

Smith, C. W., L'Heureux, J., Ness, N. F., et al.: The ACE magnetic fields experiment, Space Sci. Rev., 86(1-4), 613-632, 1998.

Song, P., Russell, C. T., Gombosi, T. I., Spreiter, J. R., Stahara, 
S. S., et al.: On the processes in the terrestrial magnetosheath, 1, Scheme development, J. Geophys. Res., 104, 22 345-22 356, 1999a.

Song, P., Russell, C. T., Zhang, X. X., Stahara, S. S., Spreiter, J. R., et al.: On the processes in the terrestrial magnetosheath, 2, Case study, J. Geophys. Res., 104, 22 357-22 374, 1999b.

Southwood, D. J. and Kivelson, M. G.: On the form of the flow in the magnetosheath, J. Geophys. Res., 97, 2873-2879, 1992.

Southwood, D. J. and Kivelson, M. G.: Magnetosheath flow near the magnetopause: Zwan-Wolf and Southwood-Kivelson theories reconciled, Geophys. Res. Lett., 22, 3275-3278, 1995.

Spreiter, J. R., Summers, A. L., and Alksne, A. Y.: Hydromagnetic flow around the magnetosphere, Planet. Space Sci., 14, 223-253, 1966.

Spreiter, J. R. and Stahara, S. S.: A new predictive model for determing solar wind-terrestrial planet interactions, J. Geophys. Res., 85, 6769-6777, 1980.

Tatrallyay, M. and Erdos, G.: The evolution of mirror mode fluctuations in the terrestrial magnetosheath Planet. Space Sci., 50(5-6), 593-599, 2002.
Tatrallyay, M., Erdos, G., Balogh, A., and Dandouras, I.: The evolution of mirror type magnetic fluctuations in the magnetosheath based on multipoint observations, Adv. Space Res., 41, 15371544, 2008.

Wu, C. C.: MHD flow past an obstacle: Large-scale flow in the magnetosheath, Geophys. Res. Lett., 19, 87-90, 1992.

Zastenker, G. N., Nozdrachev, M. N., Němeček, Z., Šafránková, J., Přech, L., Paularena, K. I., Lazarus, A. J., Lepping, R. P., and Mukai, T.: Plasma and magnetic field variations in the magnetosheath: Interball-1 and ISTP spacecraft observations, in: Interball in the ISTP Program, edited by: Sibeck, D. G. and Kudela, K., NATO Science Series, 537, 277-294, 1999.

Zastenker, G. N., Nozdrachev, M. N., Němeček, Z., Šafránková, J., Paularena, K. L., Richardson, J. D., Lepping, R. P., and Mukai, T.: Multispacecraft measurements of plasma and magnetic field variations in the magnetosheath: Comparison with Spreiter models and motion of the structures, Planet. Space Sci., 50, 601-612, 2002.

Zwan, B. J. and Wolf, R. A.: Depletion of the solar wind plasma near a planetary boundary, J. Geophys. Res., 81, 1636-1648, 1976. 